\title{
A Feared Complication of Peritoneal Dialysis Catheter Insertion
}

\author{
Moreiras Plaza M*, Fernández-Fleming F, Nájera de la Garza W, Azkárate Ramírez N and \\ Hernansanz Pérez M \\ Complexo Hospitalario Universitario de Vigo, Hospital Alvaro Cunqueiro Nephrology Department, Vigo, Spain
}

Received: November 10, 2016; Accepted: March 14, 2017; Published: March 22, 2017

*Corresponding author : Moreiras Plaza M, Complexo Hospitalario Universitario de Vigo, Hospital Alvaro Cunqueiro Nephrology Department, Vigo, Spain; E-mail: mercedes.moreiras.plaza@sergas.es

\begin{abstract}
Bowel perforation is an uncommon but potentially serious complication of PD catheter insertion. It has been reported using all of implantation techniques. The associated increased morbidity and mortality of bowel perforation after PD catheter insertion makes early recognition and prompt treatment mandatories. Nephrologists performing the procedure of catheter insertion should be well versed in diagnosing this complication. We reported a PD catheter insertion into a loop of small bowel during an open surgical PD catheter implantation and review the diagnostic criteria and management of this complication.
\end{abstract}

Key words: Peritoneal Catheter Implantation; Bowel Perforation; Peritoneal Dialysis Complication

Success in Peritoneal Dialysis (PD) is dependent on uncomplicated access to the peritoneum. The insertion of the PD catheter may be performed by percutaneous Seldinger technique, laparoscopy, fluoroscopy or open surgical approach [1]. The complications associated with the insertion of a catheter for peritoneal dialysis are usually of a minor technical nature and have little clinical implication. Rarely, however, major complications, such as intestinal, bladder, or large vessel penetration, do occur and pose a serious threat to the patient.

Bowel perforation is a recognized but uncommon complication during the insertion of PD catheters. We reported a PD catheter insertion into a loop of small bowel and review the diagnostic criteria of this complication.

\section{CASE}

A 46-year-old male with chronic renal failure due to polycystic renal disease underwent open surgical implantation of a double-cuffed self-locating PD catheter under local anesthesia and sedation. His medical history was unremarkable for prior abdominal surgeries.

The peritoneal catheter was inserted through a little right paramedian sub-umbilical incision. Subcutaneous fat dissection was carried out to reach the sheath of the abdominal rectus muscle. The anterior rectus sheath was opened, and the muscle fibers bluntly separated. The posterior sheath was then cut, and the peritoneal cavity was opened. The catheter was softly advanced (with a malleable stylet) into the peritoneal cavity by feel until the deep cuff was within the rectus muscle. After insertion of the Tenckhoff catheter into the peritoneal cavity, the peritoneum and posterior rectus sheath were closed with absorbable purse-string suture sutures. Immediate catheter testing was then performed. Although instillation of the dialyzing fluid was uneventful and first effluent was normal and showed a good drainage, the effluent from the second instillation was lightly brownish in color and contained little flecks of particulate matter.

Suspecting bowel perforation, the incision was enlarged and the catheter path was revised, revealing that the catheter tip was into a loop of small bowel that was adherent to the anterior abdominal wall (Figure). The catheter was removed and the bowel perforation was sutured. In view of the patient's good condition a new fresh catheter was inserted using the same incision.

Testing of the second catheter showed good drainage_of clear dialysate. The peritoneum and the posterior rectus sheath level were closed and the internal cuff was then secured within the rectus muscle. Free drainage of PD fluid was tested again. After repair of the anterior rectus sheath with sutures, a subcutaneous tunnel to exit site was then created. The external cuff was placed subcutaneously, at approximately $2 \mathrm{~cm}$ from the exit site. No sutures were placed at the skin exit

The patient was maintained nothing by mouth (NPO) and given intravenous antibiotics (ceftazidime and metronidazole). He was monitored closely looking for signs of peritonitis, but he remained stable clinically with normal vital signs and without evidence of peritoneal irritation. He maintained good bowel sounds and reported passage of flatus the next morning. Two days later, the patient had normal bowel movements and he was 


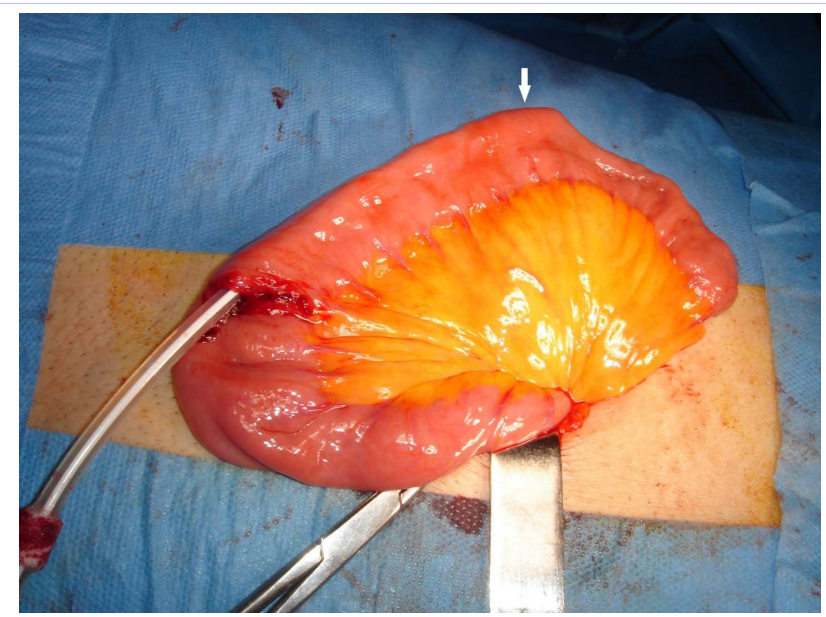

Figure 1: Small bowel catheterization: the self-locating peritoneal catheter silhouette is guessed into the loop of small bowel (arrow: silhouette of catheter tip)

started on a liquid diet that was well tolerated. Peritoneal dialysis was initiated three weeks later without any problem.

\section{Discussion}

Bowel perforation, though much feared, is an uncommon but potentially serious complication of PD catheter insertion. It has been reported using all of implantation techniques: trocars, stylet-catheters, surgical insertion or peritoneoscopic technique. Perforation of the bowel by trocar or due to passage of the styletcatheters into the peritoneum has been reported previously in the first time of peritoneal dialysis [2-5]. The incidence of visceral perforation using the dissective technique has been reported to range from $1 \%$ to $1.4 \%[1,6]$. The true incidence of bowel perforation with the peritoneoscopic technique is unknown; different studies ranged the incidence of this complication from 0 to $0.8 \%[1,7]$.

The site of injury depends on the location and direction of insertion: the cecum may be damaged if the catheter is inserted in the right iliac fossa, the sigmoid colon may be damaged if the catheter is inserted in the left iliac fossa, and if the catheter is inserted in the midline the small bowel may be perforated [3]. The bladder is at risk if care has not been taken to ensure that it is empty before the catheter is inserted [8]. It has been stated that intestinal perforation occurs more frequently in patients who have had previous abdominal operations due to the development of intra-abdominal adhesions [7]. Our patient has not history of prior abdominal surgeries, and the catheter was inserted in right paramedian sub-umbilical site, but we think that the enlarged polycystic kidneys displaced and limited the mobility of bowel loops.
Three are the criteria described for the diagnosis of perforation of the bowel during catheter insertion: retention of the dialysate, cloudy malodorous or frankly feculent fluid return (microscopy may be helpful in case of doubt), and watery diarrhea (in doubtful cases a colorant can be introduced through the catheter) [3]. In laparoscopic procedures, the visualization of bowel lumen and/or bowel contents or the return of bowel contents through the cannula is another criteria [7].

The associated increased morbidity and mortality of bowel perforation after PD catheter insertion makes early recognition and prompt treatment mandatories. Some investigators suggest that this complication should be treated with surgical intervention. However, successful conservative management of bowel perforation also has been reported $[1,3,5,9]$.

\section{Conclusion}

Although the risk of bowel perforation following PD catheter insertion is minimal, nephrologists performing this procedure should be well versed in diagnosing this complication promptly and managing the patient effectively.

\section{References}

1. Asif A. Peritoneal Dialysis Access-Related Procedures by Nephrologists. Semin Dial. 2004;17(5):98-406. doi: 10.1111/j.08940959.2004.17355.x

2. López de Novales E, Hernando Avendaño L. Risks of peritoneal catheter insertion. Lancet. 1968;291:473. doi: 10.1016/S01406736(68)92802-X

3. Simkin EP., Wright FK. Perforating injuries of the bowel complicating peritoneal catheter insertion. Lancet. 1968;1(7533):64-66.

4. Rigolosi RS, Maher JF, Schreiner GE. Intestinal Perforation during Peritoneal Dialysis. Ann Intern Med. 1969; 70(5):1013-1015. doi: 10.7326/0003-4819-70-5-1013

5. Kahn SI, Garella S, Chazan JA. Nonsurgical treatment of intestinal perforation due to peritoneal dialysis. Surg Gynecol Obstet. 1973;136(1):40-42.

6. Kai Ming Chow, Cheuk Chun Szeto, Chi Bon Leung, Bonnie Ching Ha Kwan, Wing Fai Pang, Philip Kam-tao Li. Tenckhoff catheter insertion by nephrologists: open dissection technique. Perit Dial Int. 2010; 30(5):524-527. doi: 10.3747/pdi.2009.00145

7. Asif A, Byers P, Vieira CF, Merrill D, Gadalean F, Bourgoignie JJ, Leclercq B, et al. Peritoneoscopic Placement of Peritoneal Dialysis Catheter and Bowel Perforation: Experience of an Interventional Nephrology Program. Am J Kidney Dis. 2003,42(6):1270-1274.

8. Moreiras M, Cuiña L, Rguez Goyanes G, Sobrado JA, Gil P. Inadvertent placement of a Tenckhoff catheter into the urinary bladder. Nephrol Dial Transplant. 1997;12(4):818-820.

9. Kachoie A, Safari S, Hosseinzadeh F, Vafaeimanesh J. Supportive treatment of delayed perforated colon due to peritoneal dialysis catheterization. Caspian J Intern Med. 2014; 5(3): 176-178. 